\title{
Entwicklung der \\ Arzneimittelkosten und \\ -preise in der Versorgung
}

Melanie Schröder und Carsten Telschow

Inhaltsverzeichnis

5.1 Preisentwicklung bei Arzneimitteln - 66

5.2 Neue Arzneimittel als Treiber der

Arzneimittelumsätze - 67

5.3 Von Hoch-, Höchst- und Mondpreisen - 69

5.4 Arzneimittelausgaben und Zusatzentgelte für neue Arzneimittel im Krankenhaus - 72

5.5 Hochpreisigkeit und EBIT-Margen - 75

5.6 Zusammenfassung und Fazit - 76

Literatur - 77 


\section{- Zusammenfassung}

Arzneimittelpreise von mehr als $10.000 €$, $100.000 €$ oder gar Preise jenseits der Millionengrenze waren noch vor wenigen Jahren eine absolute Ausnahme, werden aber seitdem immer häufiger zur Realität. Während es die einen gelassen sehen und auf die unproblematische Umsatzentwicklung der ambulanten Arzneimittelverordnungen hinweisen, sehen die anderen eine Gefährdung der heutigen und vor allem der zukünftigen Finanzierbarkeit. Aber welchen Einfluss haben diese hochpreisigen, meist patentgeschützten Arzneimittel auf den deutschen Arzneimittelmarkt und haben wir wirklich (k)ein Problem? Der Beitrag zeigt eine rasante Preisentwicklung insbesondere bei neuen patentgeschützten Arzneimitteln und auch eine steigende Ausgabenrelevanz von sogenannten Hochpreisern, die ihre Preisanker in immer mehr Indikationsgebieten setzen können. Zudem wird deutlich, dass die Fokussierung auf den ambulanten Markt zu kurz greift, um die Kostenauswirkung dieser neuen Arzneimittel einzuschätzen. Die Kosten der zusätzlich notwendigen Berücksichtigung für die Anwendung von Marktneueinführungen im Krankenhaus können aus der Perspektive der Zahlenden durchaus Besorgnis hervorrufen. Der Blick auf die umsatzstarken und gewinnstärksten Pharmakonzerne schließlich zeigt, dass sich deren Geschäftsfeld überwiegend auf hochpreisige und patentgeschützte Arzneimittel konzentriert.

\subsection{Preisentwicklung bei Arzneimitteln}

Wie dramatisch ist die Preisentwicklung in Deutschland, über die im Rahmen der bereits seit Jahren beklagten Mondpreise für neue Arzneimittel (Korzilius 2017; Bausch 2016; Glaeske 2016; Mühlbauer 2017; Richard 2016) immer wieder diskutiert wird, tatsächlich? Um einen Einblick in die allgemeine Preisentwicklung des Marktes zu erhalten, sind in - Abb. 5.1 die durchschnittlichen
Packungspreise der Arzneimittel seit 2010 für die Präparate des Gesamtmarktes, des Patentmarktes und der neuen patentgeschützten Arzneimittel, die innerhalb der letzten 36 Monate auf den Markt gekommen sind (neue Markteinführungen), dargestellt. Hier gehen alle verordnungsfähigen Arzneimittel als ungewichtete Mittel ein, das heißt unabhängig von ihrer Verordnungshäufigkeit. Diese Arzneimittel können sowohl von ambulant tätigen Kassenärztinnen und -ärzten verordnet als auch im Krankenhaus eingesetzt werden.

Die Darstellung ${ }^{1}$ offenbart eine rasante Preisentwicklung der Präparate des Patentmarktes und insbesondere der Preise der neuen Markteinführungen. Im Schnitt kostete die Packung eines Arzneimittels, das in den letzten 36 Monaten auf den Markt gekommen ist, 2011 noch $902 €$. 2014, als die ob ihres hohen Preises damals viel diskutierte „1.000-DollarPille“ Sovaldi ${ }^{\circledR}$ (Sanger-Katz 2014) und weitere ähnlich wirkende Arzneimittel den Markt betraten, stieg dieser Durchschnittspreis auf Werte zwischen 3.000 und $4.000 €$. Ende 2020 lag der durchschnittliche Packungspreis für neue patentgeschützte Arzneimittel dann bei $42.407 €$. Das entspricht mehr als einer Vervierzigfachung des Durchschnittspreises bei den neuen Markteinführungen in gerade einmal zehn Jahren.

Diese Entwicklung schlägt auch auf den gesamten Patentmarkt und den Gesamtmarkt durch: Anfang 2011 lag der durchschnittliche Packungspreis im Gesamtmarkt bei $180 €$, im Patentmarkt bei $1.002 €$. Zum Jahresende 2020 waren es $1.014 €$ im Gesamtmarkt und $11.775 €$ im Patentmarkt. Auch wenn die Entwicklung aufgrund der Vielzahl der im Markt befindlichen Arzneimittel nicht ganz so dynamisch ist wie bei den neuen Markteinführungen, ergibt sich hier eine Verzwölffachung im Patentmarkt und insgesamt eine Verfünffachung des Durchschnittspreises im Gesamt-

1 Diese Darstellung basiert auf den Angaben der „Preisentwicklung auf dem Arzneimittelmarkt“, die als monatliche Berichte zum freien Download unter - https://www.wido.de/publikationen-produkte/ arzneimittel-preisinformation/verfügbar sind. 


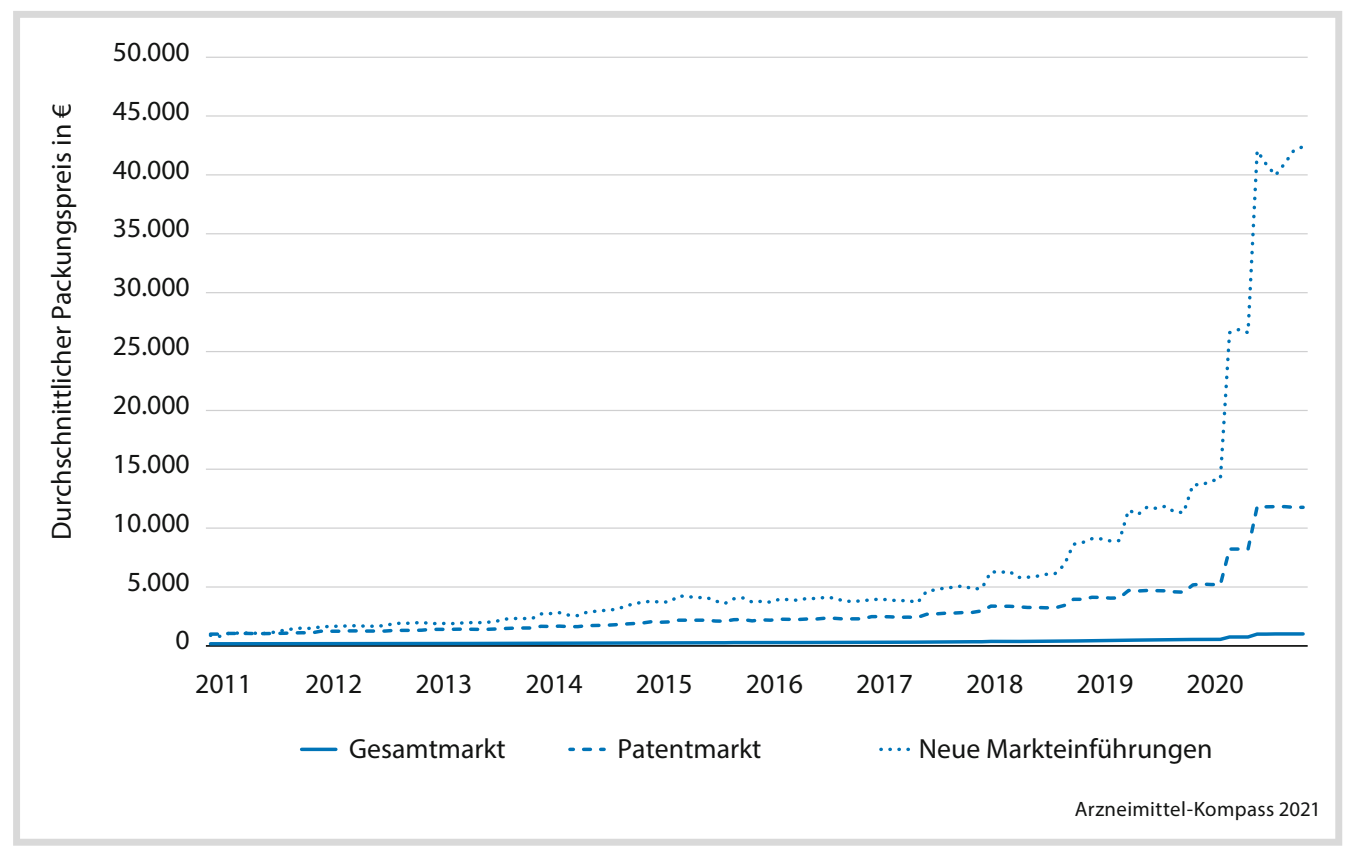

- Abb. 5.1 Entwicklung des durchschnittlichen Packungspreises nach Marktsegmenten (Quelle: GKV-Arzneimittelindex)

markt aller Arzneimittel in Deutschland - und das innerhalb der letzten zehn Jahre.

\subsection{Neue Arzneimittel als Treiber der Arzneimittelumsätze}

Wie schlagen sich diese neuen und vergleichsweise teuren Markteinführungen in den Arzneimittelumsätzen nieder und welche sind die Treiber des Umsatzwachstums? Wie aus - Abb. 5.2 ersichtlich, steigt der Umsatz im ambulanten Arzneimittelmarkt der gesetzlichen Krankenversicherung (GKV) seit 2011 von Jahr zu Jahr kontinuierlich an. 2020 erhöhte sich der GKV-Arzneimittelumsatz um 4,9\% und lag somit zuletzt bei 49,2 Mrd. $€$ (vgl. Kap. 16, Telschow et al.).

Um der Frage nachzugehen, welche Faktoren dieses Umsatzwachstum erklären, wird die Methode der Komponentenzerlegung genutzt, mit deren Hilfe die Einflussfaktoren auf das Umsatzwachstum analysiert und interpretiert werden können. Dabei werden im Wesentlichen drei Komponenten betrachtet, durch die sich die Arzneimittelumsätze im Vergleich zum Vorjahr verändern können: Der Umsatz kann steigen, weil die Preise der verordneten Arzneimittel steigen, die verordnete Menge wächst oder wenn strukturell statt günstiger teurere Arzneimittel innerhalb der Arzneimittelgruppen verordnet werden. Letzteres wird im Wesentlichen über die sogenannte Intermedikamenten-Komponente abgebildet. $^{2}$

In - Abb. 5.2 sind diese Effekte auf die Umsätze für die Jahre 2011 bis 2020 abgetragen. Es zeigt sich, dass sowohl die Preis- als auch die Mengeneffekte im Vergleich zum Intermedikamenteneffekt keine besonders starken Treiber des Umsatzwachstums darstellen.

2 Eine detaillierte Beschreibung der Komponentenzerlegung des Jahres 2020 findet sich in Kap. 16, Telschow et al. Methodische Erläuterungen finden sich in WIdO (2021). 


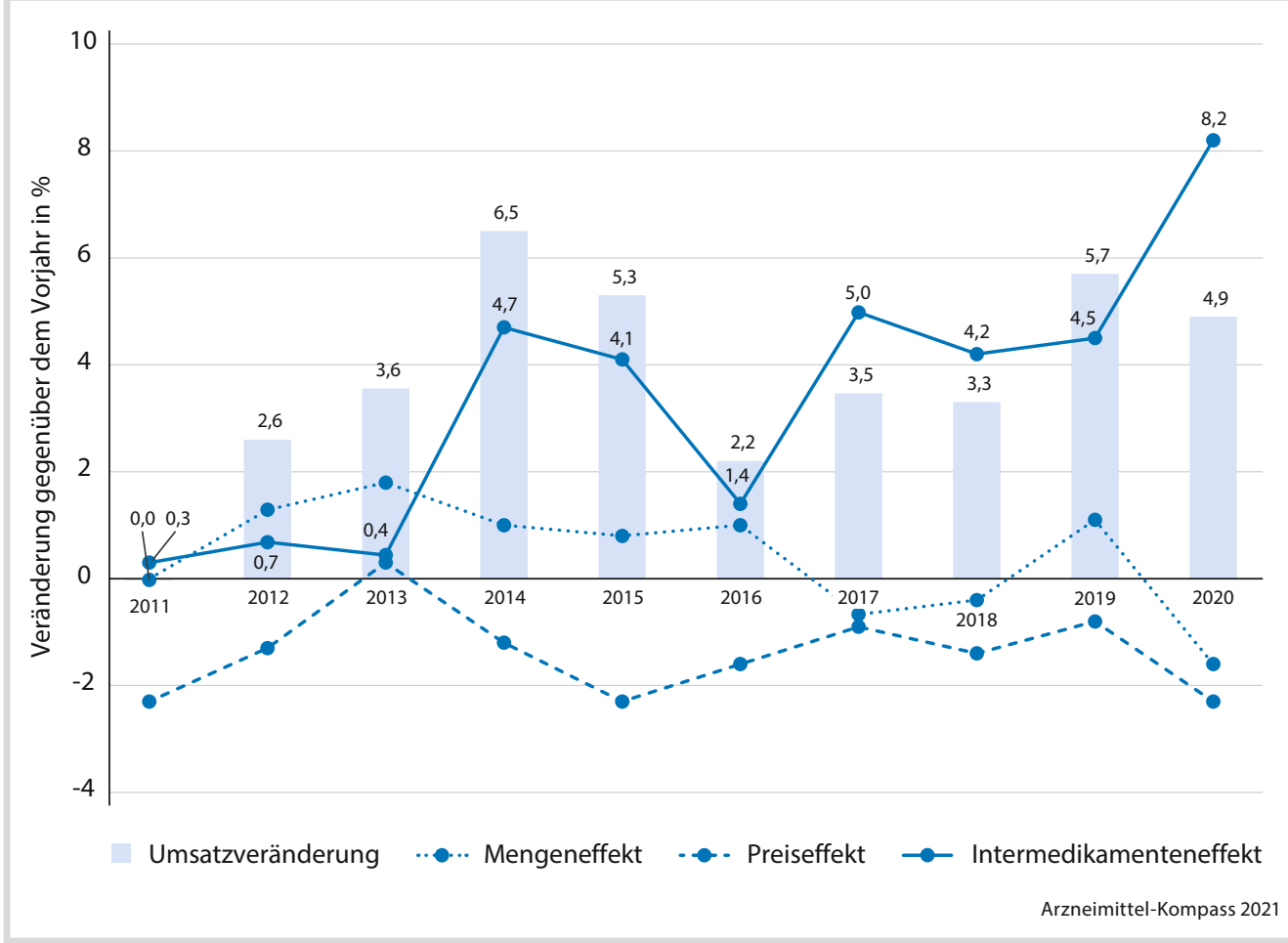

- Abb. 5.2 Umsatzwachstum und ausgewählte Komponenten des Umsatzwachstums 2011 bis 2020 (Quelle: GKVArzneimittelindex)

Der Preiseffekt lag im Beobachtungszeitraum nicht nur stets unterhalb der prozentualen Umsatzveränderung, sondern nahezu immer im negativen Bereich. Das bedeutet, dass sich die Preisentwicklung der im Markt befindlichen Präparate umsatzsenkend auswirkt. Wenn also die gleichen Präparate in der gleichen Menge wie im Vorjahr verordnet worden wären, hätte sich tatsächlich eine Umsatzsenkung ergeben. Diese allgemeine Preissenkung wird beeinflusst durch Wettbewerb, vor allen Dingen im generikafähigen Markt, und flankiert von gesetzlichen Maßnahmen wie Festbeträgen, den AMNOG-Erstattungsbeträgen, dem Preismoratorium und 2020 einer Mehrwertsteuerabsenkung im zweiten Halbjahr.

Auch der Mengeneffekt, der die Umsatzveränderung misst, die sich allein durch eine Zunahme der Arzneimittelverordnungen ergeben hat, kann nicht als Treiber identifiziert werden. Er bewegt sich im betrachteten Zeitraum zwischen $-2 \%$ und $+2 \%$ und liegt stets unterhalb des gesamten Umsatzwachstums.

Dagegen zeigt der Intermedikamenteneffekt einen besonderen Einfluss auf die Umsatzveränderung, die sich allein durch eine strukturell veränderte Verordnung von Arzneimitteln innerhalb der Arzneimittelgruppen ergeben hat. Es handelt sich hier um einen Wanderungseffekt, der oft auch als Verschiebung der Verordnungen hin zu anderen Präparaten der gleichen Arzneimittelgruppe beschrieben wird. In bestimmten Bereichen setzen sich einige Arzneimittel immer stärker im Markt durch, sei es durch wissenschaftliche Erkenntnisse, durch Empfehlungen in ärztlichen Therapieleitlinien, durch Vorgaben der Selbstverwaltung im Gesundheitssystem oder durch entsprechende werbewirksame Maßnahmen der pharmazeutischen Hersteller. Der In- 
termedikamenteneffekt ist also auch ein Ausdruck des Therapiewandels, der sich in meist steigenden Umsätzen niederschlägt.

Das wird exemplarisch deutlich an der starken Bewegung des Intermedikamenteneffekts der Jahre 2014 bis 2016: 2014 betrat die ,1.000-Dollar-Pille“ Sovaldi ${ }^{\circledR}$ den Markt, die erstmals eine Heilung von Hepatitis-C ermöglichte. ${ }^{3}$ Die starke Verordnungsausweitung dieses Medikaments und nachfolgender, vergleichsweise sehr teurer Arzneimittel führte in dieser Gruppe zu einem sehr starken Intermedikamenteneffekt von 53,9\% in diesem Jahr, der sich auch im Gesamtmarkt niederschlug (• Abb. 5.2). Mit diesen Arzneimitteln konnte eine Heilung bei einem beträchtlichen Anteil der Patientinnen und Patienten mit dieser schwerwiegenden Krankheit erreicht werden, sodass folglich immer weniger therapiert werden mussten. Nachdem einige ähnliche Arzneimittel den Markt der Hepatitis-CTherapeutika betraten, liegt - vor allem durch den Produktwettbewerb - seit 2016 der Intermedikamenteneffekt dieser Gruppe stets im negativen Bereich. Es kann abgeschätzt werden, dass zwischen 2014 und 2016 insgesamt mehr als 46.000 Hepatitis-C-Patient:innen therapiert werden konnten, was mit Nettokosten von mehr als 2,6 Mrd. $€$ verbunden war.

Während der Intermedikamenteneffekt damals maßgeblich von dem Trend in einer Indikation beeinflusst wurde, wird dieser Effekt üblicherweise von verschiedenen Arzneimittelgruppen getragen. Insgesamt ist seit mehreren Jahren zu beobachten, dass die Therapiefortschritte aber eben auch ein starkes Umsatzwachstum in mehreren Arzneimittelgruppen durch deutlich ansteigende Intermedikamenteneffekte hervorbringen. Neben den in der Krebsbehandlung eingesetzten antineoplastischen Mitteln, die in den letzten Jahren stets einen Intermedikamenteneffekt im zweistellig positiven Bereich aufwiesen, liegt 2020 auch

3 Bei Anwendung der möglichen Kombinationen direkt wirkender antiviraler Mittel werden Ansprechraten von ca. 50-90 \% mit geringen Rückfallraten erreicht, abhängig vom klinischen Bild und dem Virus-Genotyp (Sarrazin et al. 2018). ein großes Umsatzwachstum im Bereich der zur Behandlung von Augenkrankheiten eingesetzten Ophthalmika, der Immunsuppressiva, die üblicherweise gegen Autoimmunerkrankungen wie Multiple Sklerose, Rheuma oder Psoriasis eingesetzt werden, und der antithrombotischen Mittel, die zur Prophylaxe von Schlaganfällen und Herzinfarkten zum Einsatz kommen, vor (siehe Kap. 16, Telschow et al. und WIdO 2021).

Gleichzeitig sind die Mengen- und Preiseffekte bei den genannten Gruppen zwar positiv, aber gegenüber dem Intermedikamenteneffekt weniger ausgeprägt. Der Umsatzanstieg erklärt sich also weitgehend über die verstärkte Verordnung teurerer Präparate, insbesondere durch die Einführung zahlreicher neuer hochpreisiger Arzneimittel in den letzten Jahren. Zudem fallen vermehrt auch Therapien gegen weitere chronische Erkrankungen in dieser Dynamik auf, wenn neue und oft sehr spezifisch wirkende Arzneimittel auf den Markt gebracht werden. So wurden in den vergangenen Jahren beispielsweise spezielle Antikörper zur Behandlung von weit verbreiteten Fettstoffwechselstörungen oder Migräne auf den Markt gebracht, die diese Therapien insgesamt erheblich verteuert haben. Anders als bei den Hepatitis-C-Arzneimitteln, die eine Heilung in Aussicht stellen können, bleibt es aber bei diesen chronischen Erkrankungen auch mit den neueren Wirkstoffen meist bei einer dauerhaften Behandlung, sodass diese die Umsätze, auch durch neu hinzugekommene $\mathrm{Pa}-$ tient:innen, dauerhaft steigen lassen.

\subsection{Von Hoch-, Höchst- und Mondpreisen}

Auch wenn immer wieder von Mondpreisen (Korzilius 2017; Bausch 2016; Glaeske 2016; Mühlbauer 2017; Richard 2016) oder Hochpreisen (Deutsche Apothekerzeitung 2016) berichtet wird, so fehlt aktuell eine allgemeine Definition eines „Hochpreisers“. Beispielsweise definiert die Deutsche Apothekerzeitung 
(2016) einen Hochpreis ab einem Packungspreis von $1.200 €^{4} \mathrm{bzw}$. verweist auf eine Definition des Datendienstleisters Insight Health mit einem Packungspreis von über $4.000 €$. Sie zeigen, dass diese Arzneimittel nicht nur in der Menge, sondern auch in Umsatzanteilen steigen (Deutsche Apothekerzeitung 2016). Im AMNOG-Report der DAK wird von extrem teuren Therapien bei einem Jahrestherapiepreis von $100.000 €$ berichtet (Greiner et al. 2020). Während diese hohen Jahrestherapiekosten in den Jahren 2011 bis 2014 nur jedes achte AMNOG-bewertete Arzneimittel betrafen, sei dies zwischen 2015 und 2018 bereits für jedes vierte AMNOG-Arzneimittel der Fall gewesen (Greiner et al. 2020). Lohmüller et al. betrachten das oberste Perzentil der durchschnittlichen Kosten je Tagesdosis, um ebenfalls einen Einblick in die Entwicklung der Hochpreisigkeit zu nehmen und zeigen, dass der Preis je Tagesdosis für das oberste Perzentil, das rund 80 Arzneimittel betrifft, in rund zehn Jahren um $150 \%$ gestiegen ist (Lohmüller et al. 2019).

Da aktuell keine allgemeingültige Definition vorliegt, werden im Folgenden drei Grenzwerte betrachtet, damit ein differenzierter Blick auf die Entwicklung der Hochpreisigkeit ermöglicht werden kann. In einer ersten Gruppe befinden sich Arzneimittel, deren Packungspreise bei $1.000 €$ und mehr liegen 2010 traf dies auf 9,0\% aller ambulant verordneten Wirkstoffe zu. Bei diesen war mindestens eine Packung in diesem Preisbereich vertreten - 2020 waren es bereits $18,7 \%$. In der zweiten Gruppe sind Arzneimittel, die mindestens 5.000€ kosten; 2010 waren dies noch $1,3 \%$ der Wirkstoffe und 2020 bereits $5,7 \%$. In der dritten Gruppe befinden sich Arzneimittel mit Packungspreisen von $10.000 €$ und mehr, die 2010 mit lediglich 0,5\% noch eine Ausnahmeerscheinung waren und 2020 auf $2,7 \%$ der ambulant verordneten Wirkstoffe

4 Oft wird hier auf die Deckelung des Großhandelszuschlags nach Arzneimittelpreisverordnung abgestellt, wonach ein (aufschlagsgedeckeltes) Arzneimittel ab einem Endpreis von $1.575 €$ als Hochpreiser bezeichnet wird.
Tab. 5.1 Anzahl der Wirkstoffe und der verordneten Tagesdosen sowie Bruttoumsätze im Gesamtmarkt und bei hochpreisigen Arzneimitteln 2010 und 2020 sowie deren Anteile am Gesamtmarkt

\begin{tabular}{l|l}
2010 & 2020
\end{tabular}

Anzahl der Wirkstoffe

Gesamt 1.717 1.914

Preis $\geq 1.000 €$ 154 357 $(9,0 \%)$ $(18,7 \%)$

Preis $\geq 5.000 €$ 22 109 $(1,3 \%)$ $(5,7 \%)$

Preis $\geq 10.000 €$ 9 51 $(0,5 \%) \quad(2,7 \%)$

Anzahl Verordnungen in Mio.

\begin{tabular}{l|r|r}
\hline Gesamt & 626,3 & 684,2 \\
\hline Preis $\geq 1.000 €$ & 2,1 & 7,5 \\
& $(0,3 \%)$ & $(1,1 \%)$ \\
\hline Preis $\geq 5.000 €$ & 0,0 & 0,5 \\
& $(0,0 \%)$ & $(0,1 \%)$ \\
\hline Preis $\geq 10.000 €$ & 0,0 & 0,1 \\
& $(0,0 \%)$ & $(0,0 \%)$
\end{tabular}

Bruttoumsatz in Mio. $€$

\begin{tabular}{|l|r|r|}
\hline Gesamt & $29.724,7$ & $49.156,0$ \\
\hline Preis $\geq 1.000 €$ & $5.064,7$ & $20.871,5$ \\
& $(17,0 \%)$ & $(42,5 \%)$ \\
\hline Preis $\geq 5.000 €$ & 320,9 & $3.850,7$ \\
& $(1,1 \%)$ & $(7,8 \%)$ \\
\hline Preis $\geq 10.000 €$ & 95,9 & $1.468,2$ \\
& $(0,3 \%)$ & $(3,0 \%)$
\end{tabular}

Quelle: GKV-Arzneimittelindex

Arzneimittel-Kompass 2021

angestiegen sind (- Tab. 5.1). Anhand der steigenden Präsenz dieser Wirkstoffe zeigt sich: Hochpreisigkeit ist nicht mehr nur auf sehr wenige Präparate oder Wirkstoffe begrenzt. Beinahe bei jedem fünften eingesetzten Wirkstoff kommen Packungspreise von $1.000 €$ oder mehr vor.

Es zeigt sich zudem, dass sich die Verordnungsrelevanz (Verordnungsanteil dieser 
Wirkstoffe an allen Verordnungen) zwar immer noch auf einem sehr niedrigen Niveau befindet - alle Hochpreiser decken 2020 lediglich 1,1\% der Versorgung nach Verordnungen ab - die Umsatzanteile (Umsatzanteil dieser Arzneimittel am Gesamtumsatz) steigen auf der anderen Seite aber deutlich an. So entfallen auf die hochpreisigen Arzneimittel, die $1.000 €$ und mehr kosten, 2020 bereits $43 \%$ des Gesamt-Bruttoumsatzes, während es 2010 noch $17 \%$ waren. 2020 entspricht das einem Umsatz von 20,9 Mrd. €, der hauptsächlich, aber nicht ausschließlich, durch Präparate des Patentmarktes gekennzeichnet ist. Mehr als drei Viertel entfallen auf patentgeschützte Arzneimittel, der Rest entfällt zum größten Teil auf den biosimilarfähigen Markt, in dem trotz des Preiswettbewerbs immer noch hohe Preise an der Tagesordnung sind.

Eine ähnlich starke Dynamik wird auch bei den Umsatzanteilen der beiden anderen Gruppen deutlich. Von $1 \%$ im Jahr 2010 wuchs der Umsatzanteil der „Hochpreiser“ zu $5.000 €$ und mehr um fast das Achtfache auf $8 \%$ im Jahr 2020. Absolut ist damit der Umsatz in dieser Preiskategorie von 0,32 Mrd. $€$ auf 3,85 Mrd. $€$ angestiegen. Versorgungsseitig ist der Anteil von $0,007 \%$ auf $0,078 \%$ im betrachteten Zeitraum gestiegen.

Dabei finden sich nicht alle Arzneimittel mit einem Packungspreis von 5.000€ oder mehr im Bereich der Krebserkrankungen, wie man angesichts der Präsenz der Onkologika in der Hochpreisdiskussion und der starken Umsatzentwicklung (siehe $>$ Kap. 6, Ludwig und Vokinger) annehmen könnte. Der Umsatzanteil dieser Gruppe an allen Hochpreisumsätzen umfasst zwar auch 2020 immer noch 36\% und bleibt damit über die letzten elf Jahre relativ konstant (• Abb. 5.3). Allerdings verteilen sich die weiteren Umsätze 2020 auf viel mehr Indikationsgruppen als noch 2010. Auch wenn die Anteile der hinzugekommenen Indikationsgruppen nicht besonders hoch sind und alle unter einem Anteil von 10\% liegen, entfalten sie trotzdem eine Wirkung: Wenn Hochpreisigkeit Einzug in immer mehr Indikationsgruppen (aktuell sind es 9 von 18) hält, beeinflusst sie nicht nur die aktuellen, sondern durch den Effekt der so gesetzten Preisanker auch die zukünftigen Therapiepreise, da sich die Arzneimittelpreise auch an den Preisen der existierenden Vergleichstherapien orientieren. Das bekannteste Beispiel ist hier sicherlich Zolgensma ${ }^{\circledR}$, bei dem der stolze Preis von 1,9 Mio. $€$ pro Heilung versprechender Einmaltherapie mit dem Preis einer zehnjährigen Therapie mit dem - ebenfalls sehr hochpreisigen - Konkurrenzprodukt Spinraza ${ }^{\circledR}$ begründet wird (Kerpel-Fronius et al. 2020). Aber auch auf anderen Gebieten wie der Enzymersatztherapie und der Multiplen Sklerose dreht sich die Preisspirale in den letzten Jahren besonders schnell.

Einen positiven Trend verzeichnen wohl auch die Kosten für die Entwicklung neuer Arzneimittelwirkstoffe, wie eine aktuelle Metaanalyse zeigt, die auf 22 Studien basiert (Schlander et al. 2021). Statt eines Wertes geben die Autor:innen einen Korridor für Entwicklungskosten zwischen 161 Mio. und 4,54 Mrd. Dollar an, da die Studien durch große Heterogenität gekennzeichnet seien. Es wird zum einen deutlich, dass es wohl keine eindeutige Definition für Entwicklungskosten gibt und Einflussfaktoren unterschiedlich berücksichtigt werden. Zum anderen mangle es einigen Studien wohl an Datentransparenz, was die Replizierbarkeit und Überprüfbarkeit der Untersuchungen erschwere. Dies sei vor allen Dingen bei den viel zitierten Studien (wie bspw. DiMasi et al. 1991, 2003, 2016) der Fall, die durch eine Nähe zur pharmazeutischen Industrie gekennzeichnet seien und eher höhere Entwicklungskosten auswiesen (Schlander et al. 2021). Seriöse und nachvollziehbare Abschätzungen der F \& E-Kosten, die auf transparenten Daten basieren, sollten in jedem Fall aber auch eine Information über den Anteil der öffentlichen Finanzierung beinhalten. 


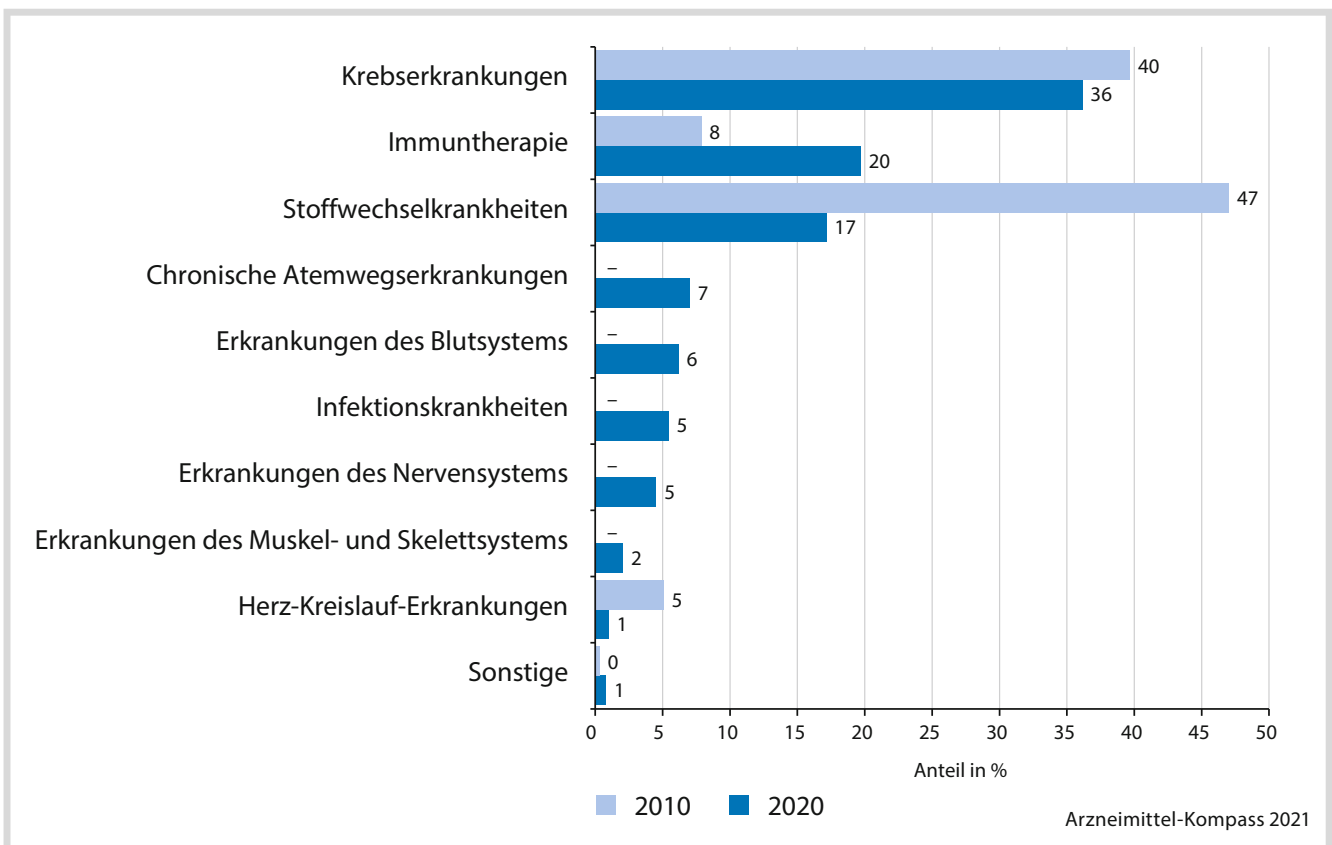

- Abb. 5.3 Umsätze der Präparate mit Packungspreisen von 5.000€ und mehr nach Indikationsgruppen 2010 und 2020 (Quelle: GKV-Arzneimittelindex)

\subsection{Arzneimittelausgaben und Zusatzentgelte für neue Arzneimittel im Krankenhaus}

Während einerseits eine starke Dynamik bei der Entwicklung der Preise, insbesondere für neue Arzneimittel (• Abb. 5.1), zu sehen ist, vollzieht sich das Umsatzwachstum des Arzneimittelmarktes insgesamt vergleichsweise moderat (• Abb. 5.2). Vor diesem Hintergrund fällt die Bewertung der Situation durch die Akteur:innen unterschiedlich aus: Einerseits herrscht die Sorge um die Bezahlbarkeit der Gesundheitsversorgung auch mit Arzneimitteln. Andererseits wird die Entwicklung der Arzneimittelausgaben von Seiten der pharmazeutischen Industrie als unauffällig beschrieben, gerade wenn der konstant hohe Anteil der ambulanten Arzneimittelausgaben an allen Gesundheitsausgaben als Indikator gewählt wird (siehe $>$ Kap. 14, Steutel; Cassel und Ulrich 2020).

Dass diese Argumentation und die Fokussierung auf ambulante Arzneimittelumsätze in Bezug auf die Umsatzwirkung neuer Arzneimittel vor dem Hintergrund der rasanten Entwicklungen bei den neuen Arzneimitteltherapien etwas zu kurz greift, soll hier exemplarisch skizziert werden. Unter den neuen Markteinführungen befinden sich zunehmend Arzneimittel, die aufgrund ihrer Komplexität nur in speziellen Zentren und/oder ausschließlich im Krankenhaus angewendet werden, wie beispielsweise bestimmte Zell- und Gentherapien oder Arzneimittel für neuartige Therapien (sogenannte ATMPs). Wenn diese komplexen Arzneimittel im Krankenhaus angewendet werden, fallen die Ausgaben auch nur dort an und bleiben bei der Betrachtung der (ambulanten) Arzneimittelausgaben unberücksichtigt. Der Gesetzgeber hat hier bereits im Jahr 2017 reagiert, indem das GKV-Arzneimittelversorgungsstärkungsgesetz (AMVSG) diese 
Anwendungen berücksichtigt und die Geltung des zwischen dem pharmazeutischen Unternehmer und dem GKV-Spitzenverband ausgehandelten Erstattungsbetrages auf den stationären Sektor ausweitet (siehe Kap. 17, Erdmann et al.). Auch die Umsatzschwelle von 50 Mio. $€$ pro Jahr, die zu einer vollen Nutzenbewertung von Orphan Drugs führt, wurde gemäß dem Gesetz für mehr Sicherheit in der Arzneimittelversorgung (GSAV) 2019 auf die stationären Umsätze erweitert.

Im stationären Setting wird der Einsatz von Arzneimitteln jedoch unterschiedlich abgerechnet. Zunächst werden aufgrund der Fallpauschalen keine separaten Arzneimittelausgaben wie im ambulanten Bereich erfasst; mit der Fallpauschale ist im Regelfall auch der Einsatz von Arzneimitteln abgegolten. Damit ist eine gezielte Auswertung der verschiedenen Kosten einzelner Positionen im Bereich der Apparatemedizin oder Arzneimittel kaum möglich. Ausnahmen bilden allerdings die sogenannten Zusatzentgelte, welche beispielsweise für teure Arzneimittel und aufwendige Prozeduren zusätzlich zu der Fallpauschale vergütet werden. Dabei ist zu unterscheiden zwischen bewerteten Zusatzentgelten, die nach einem bundesweit einheitlich kalkulierten Preis vergütet werden, und hausindividuellen Zusatzentgelten, für die auf Einzelhausebene aufgrund einer unzureichenden Datenbasis für eine bundesweite Preiskalkulation Entgelte gemäß $\S 6$ Abs. 2 KHEntgG oder $\S 6$ Abs. $2 a$ KHEntgG vereinbart werden müssen. Bei den hausindividuellen Zusatzentgelten handelt es sich meist um seltene, neuartige und/oder kostenintensive Therapien. In Ermangelung von GKV-bezogenen Ausgaben werden für diesen Beitrag die Ausgaben für AOK-Versicherte für hausindividuell vereinbarte Zusatzentgelte der Krankenhäuser ${ }^{5}$, die in Zusammenhang mit Arzneimitteln stehen, genutzt. Auf Basis der AOK-Arzneimittelumsätze in der ambulanten

5 Siehe Leclerque und Mostert (2021) für eine Beschreibung der Leistungsentwicklung im Zusatzentgelte-Bereich. und stationären Versorgung werden die Umsätze der GKV abgeschätzt.

Damit diese Ergebnisse vor dem Hintergrund der „Hochpreisigkeit“ interpretiert werden können, wird der Warenkorb für die ambulanten und stationären Arzneimittelumsätze auf hochpreisige Arzneimittel eingegrenzt. Dabei wird ausschließlich auf Arzneimittel mit neuen Wirkstoffen fokussiert, die in den letzten drei Jahren zugelassen wurden. Im Jahr 2015 beispielsweise werden alle neuen Wirkstoffe betrachtet, die seit 2013 auf den Markt gekommen sind. Damit die ambulanten und stationären Warenkörbe vergleichbar sind, werden zudem nur die neuen Arzneimittel auch in den ambulanten Arzneimittelumsätzen betrachtet, für die Zusatzentgelte individuell vereinbart wurden.

Seit 2011 haben sich die hausindividuellen Zusatzentgelte, für die in den Entgeltschlüsseln ein Hinweis auf die Gabe oder Anwendung der Wirkstoffe des Warenkorbs gefunden werden konnte, kontinuierlich und seit 2016 sehr stark erhöht (- Abb. 5.4). Der leichte Rückgang im Jahr 2020 ist damit zu erklären, dass der Wirkstoff Nusinersen (Spinraza ${ }^{\circledR}$ ) nach drei Jahren im Markt nicht mehr als „neu“ galt und somit für 2020 aus dieser Betrachtung herausfiel. Setzt man diese abgerechneten hausindividuellen Zusatzentgelte für die stationäre Versorgung mit neuen Arzneimitteln ins Verhältnis zu den ambulanten Umsätzen für diese neuen Arzneimittel, so zeigt sich seit 2016 auch ein starker Anstieg dieses Verhältnisses. Vor zehn Jahren lag ein „Aufschlag“ auf die ambulanten Arzneimittelumsätze, um die gesamten Arzneimittelumsätze für die neuen Arzneimittel abzuschätzen, bei weniger als $10 \%$. 2020 liegt dieser „Aufschlag" auf die ambulanten Arzneimittelumsätze bereits bei über $50 \%$.

Wird dieses auf den Arzneimittelausgaben (hausindividuelle Zusatzentgelte und ambulante Umsätze) der AOK-Versicherten basierende Verhältnis für die GKV-Arzneimittelkosten des gleichen Warenkorbes übertragen, zeigt sich: Die ambulanten GKV-Arzneimittelumsätze für die neuen Arzneimittel, 


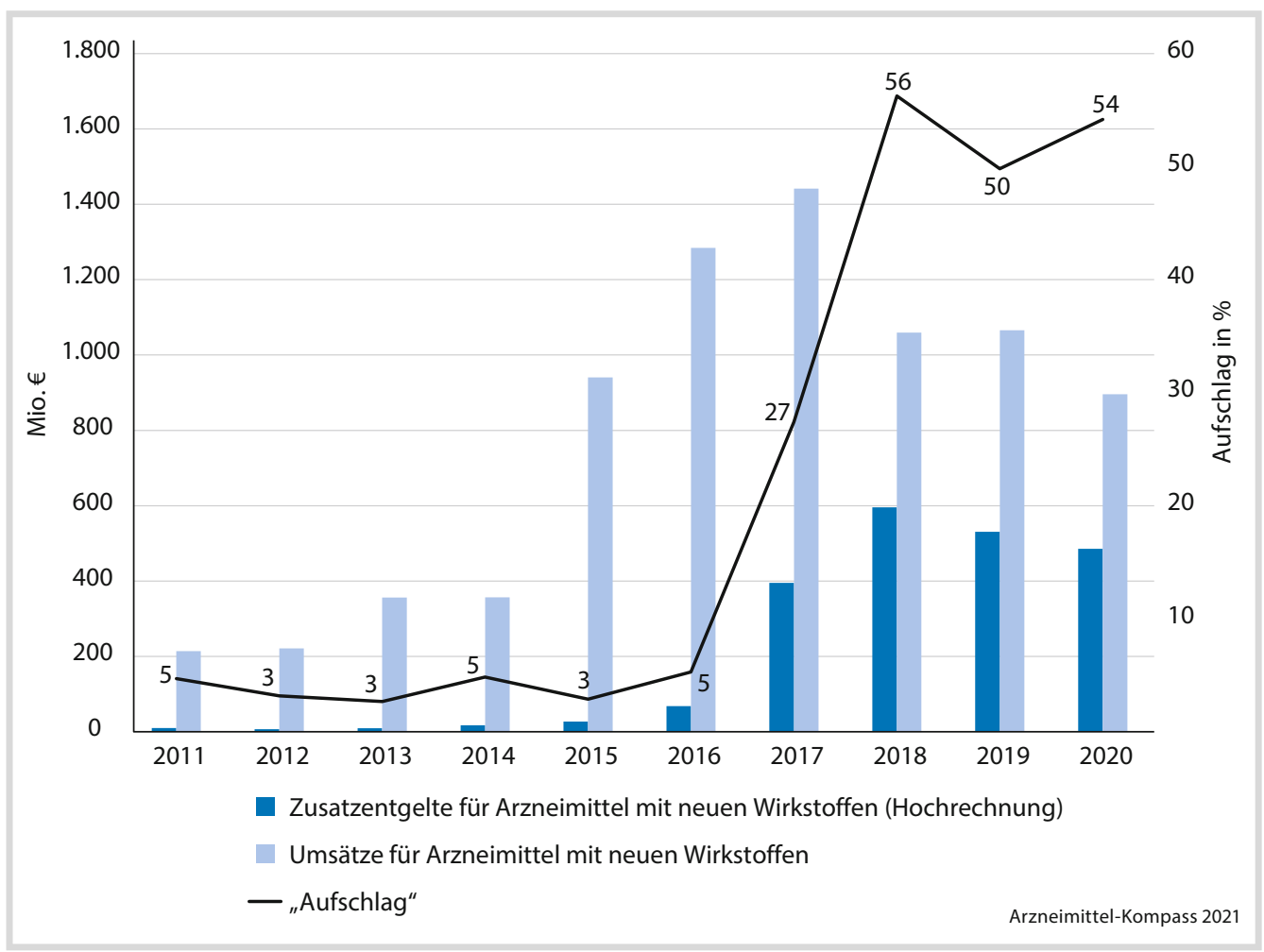

- Abb. 5.4 Gemeinsame Betrachtung der hochgerechneten Zusatzentgelte (stationär) und der Arzneimittelumsätze (ambulant) neuer Arzneimittel (Quelle: GKV-Arzneimittelindex)

die auch im Rahmen von hausindividuellen Zusatzentgelten im Krankenhaus abgerechnet werden, betrugen 2019 in Summe 1,1 Mrd. €. Es kann davon ausgegangen werden, dass sich die GKV-Ausgaben unter Einbeziehung der abgerechneten hausindividuellen Zusatzentgelte auf 1,6 Mrd. $€$ erhöhen würden.

Dabei ist zu berücksichtigen, dass es sich bei dieser Summe nur um Arzneimittel mit neuen Wirkstoffen handelt, welche über die hausindividuellen Zusatzentgelte abgerechnet wurden. Andere hochpreisige Arzneimittel oder Arzneimittel des Patentmarktes, die im ambulanten Markt nicht zum Einsatz kommen, sowie Arzneimittel, die nicht über hausindividuelle Zusatzentgelte abgerechnet werden, sondern mittlerweile in die bundesweite Kalkulation oder bepreiste Zusatzentgelte übergegangen sind und damit über Fallpauschalen abgerechnet werden, fehlen in dieser Betrachtung.

Es zeigt sich somit die Notwendigkeit, bei der Betrachtung der Arzneimittelausgaben für neue Wirkstoffe auch die stationäre Versorgung mit zu berücksichtigen. Mit weiteren Zulassungen von ATMPs und Gentherapien, die in den nächsten Jahren erwartet werden (Haas et al. 2019), sollte bei der Ermittlung der Arzneimittelkosten der Ort der Therapien - ob Arztpraxis oder Krankenhaus - keine Rolle spielen. Eine integrierte Betrachtung der Kosten in der ambulanten und stationären Versorgung scheint daher notwendig zu sein. Entsprechende Instrumentarien, die in der digitalen Zeit mehr Transparenz über die Arzneimittelkosten in der stationären Versorgung möglich machen, sind aufwandsarm aufzubauen. Die Abrechnungsdaten der Kran- 
kenhäuser müssten dafür um die digital vorliegenden Arzneimittelverordnungen und -kosten ergänzt werden. Die Preise, die im stationären Bereich meist bilateral zwischen Hersteller und Krankenhäusern oder deren Einkaufsgemeinschaften vereinbart werden, sind zudem vertraulich. In diesem gänzlich intransparenten Gefüge fehlt derzeit die Möglichkeit, reale und abgerechnete Preise abzugleichen.

\subsection{Hochpreisigkeit und EBIT- Margen}

Im Zusammenhang mit der Hochpreisigkeit von Arzneimitteln werden auch immer wieder die großen Gewinne der Pharmaindustrie thematisiert, die sie unter anderem durch ihre Marktmacht mit neuen, patentgeschützten Arzneimitteln erzielen kann (Morgan et al. 2020).

Die Gewinnmargen (EBIT-Margen), die von der Unternehmensberatung Ernst \& Young jährlich für die weltweit Top 21Pharmaunternehmen veröffentlicht werden, lagen im Jahr 2020 durchschnittlich bei $25,7 \%$ (EY 2021). Dies ist zunächst Ausweis einer extrem profitablen Branche, denn selbst die Informationstechnologie mit einer durchschnittlichen EBIT-Marge von 18,8\% (EY 2019) liegt deutlich darunter. In Deutschland erwirtschafteten diese Top 21-Unternehmen 2020 bereits mehr als die Hälfte der GKV-Nettokosten und sicherten damit rund $26 \%$ der GKVVersorgung (vgl. $>$ Kap. 16, Telschow et al.).

Doch womit erzielen diese Top 21Pharmaunternehmen ihre Umsätze in Deutschland? Sind es tatsächlich hauptsächlich patentgeschützte bzw. hochpreisige Arzneimittel? Während die Top 21-Pharmaunternehmen rund $53 \%$ der gesamten Nettokosten im Gesamtmarkt auf sich vereinen, ist ihre Umsatzrelevanz (nach Nettokosten) im Bereich der patentgeschützten Arzneimittel und Hochpreis-Arzneimittel noch deutlich höher: Diese Pharmaunternehmen decken in Deutschland bei den Hochpreisern (Arzneimittel mit ei-

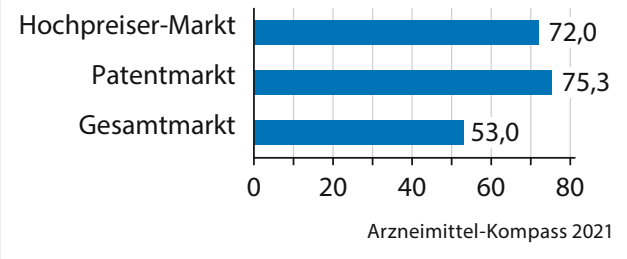

- Abb. 5.5 Nettokostenanteil der weltweit Top 21-Pharmaunternehmen im Gesamtmarkt und in ausgewählten Marktsegmenten 2020 (Quelle: GKV-Arzneimittelindex)

nem mittleren Preis von mindestens $1.000 €$ ) mit Nettokosten von 20,0 Mrd. $€ 2020$ einen Anteil von $72,0 \%$ ab - anteilig an allen Nettokosten patentgeschützter Arzneimittel von 23,1 Mrd. € sogar 75,3\% (• Abb. 5.5).

In - Abb. 5.6 werden die Umsatz- und Versorgungsanteile der Top 21 und allen anderen Unternehmen in den interessierenden Teilmärkten gegenübergestellt und ins Verhältnis zum Gesamtmarkt gesetzt. Hier zeigt sich, dass $31,2 \%$ der Nettokosten des Gesamtmarktes auf Hochpreisarzneimittel der Top 21 entfallen; bei patentgeschützten Arzneimitteln sind es sogar $37,6 \%$. Verordnungsseitig sind diese Marktsegmente mit einem Anteil von $0,8 \%$ bei Hochpreisern oder $4,4 \%$ mit patentgeschützten Arzneimitteln jedoch eher irrelevant.

Es deutet sich an, dass die hohen Gewinne der Top 21-Pharmaunternehmen insbesondere im Patentmarkt und mit hochpreisigen Arzneimitteln erzielt werden; diese kommen jedoch nur einer vergleichsweise kleinen Gruppe von Arzneimittelpatient:innen zugute, was die geringe Versorgungsabdeckung deutlich macht. Die Fokussierung der pharmazeutischen Industrie insbesondere auf Patent- oder Hochpreisarzneimittel mit hohen Umsatzanteilen trotz einer geringen Versorgungsabdeckung wird ermöglicht, da patentgeschützte Arzneimittel allenfalls einem begrenzten Wettbewerb ausgesetzt sind und die Preise von den pharmazeutischen Unternehmen weitestgehend frei gestaltet werden können. Die kaum gebremste Preisdynamik bei Patentarzneimit- 

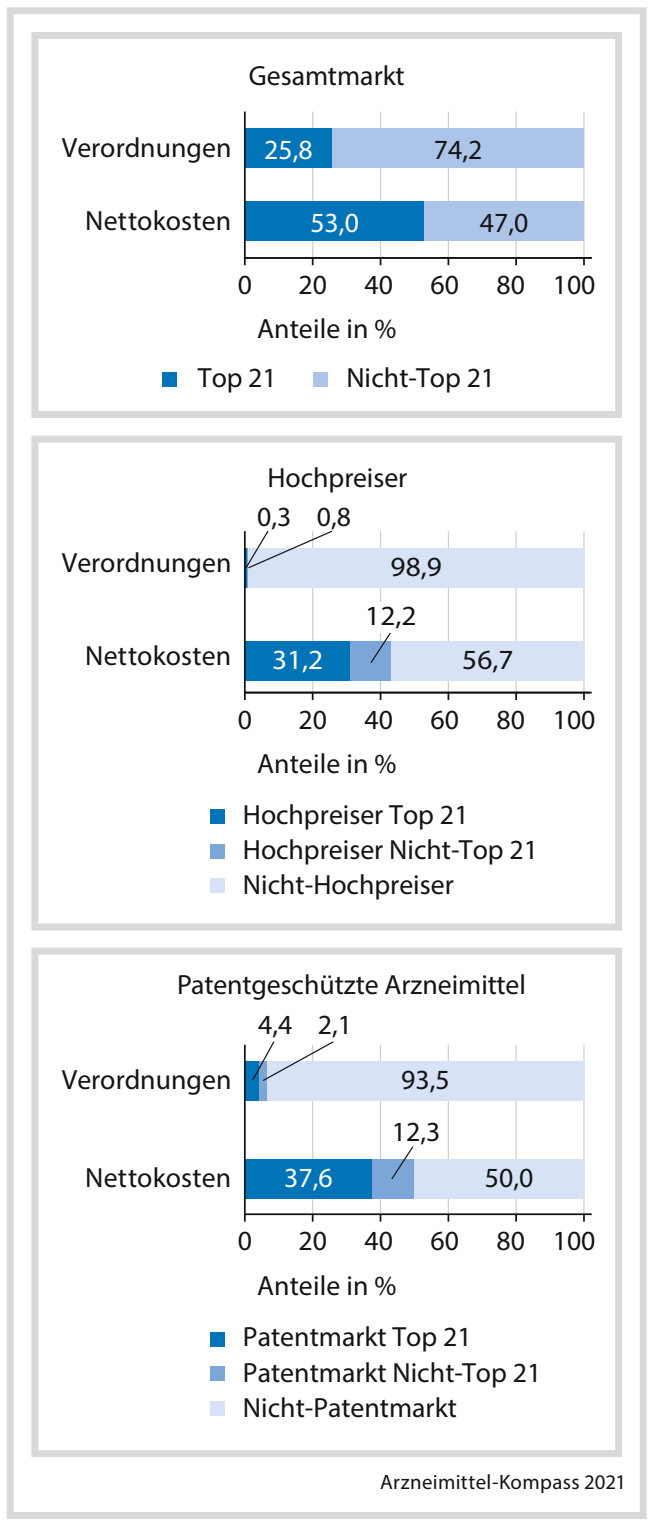

- Abb. 5.6 Top 21-Pharmaunternehmen mit ihren Nettokostenanteilen und Verordnungsanteilen im Gesamtmarkt und in ausgewählten Marktsegmenten 2020 (Quelle: GKV-Arzneimittelindex)

teln, die auf immer mehr Indikationsgebiete übergreift, kann durchaus Besorgnis um die zukünftige Finanzierbarkeit der Arzneimittelversorgung hervorrufen. Es erscheint zwingend notwendig, dass ein Ausgleich zwischen den Gewinnerzielungsabsichten der Unternehmen und der nachhaltigen Finanzierbarkeit der Versorgung in Deutschland, Europa und der Welt gefunden werden muss. Nur so kann auch zukünftig der Zugang zu und die Finanzierung von neuen Arzneimitteln sichergestellt werden.

\subsection{Zusammenfassung und Fazit}

Die Preisentwicklung in der ambulanten Versorgung ist rasant. Gründe sind in den hohen Werten, die sich aus strukturellen Effekten ergeben, zu suchen, die dokumentieren, dass immer teurere Arzneimittel in der Therapie eingesetzt werden. Würde nur die Verordnungsumlenkung auf andere, meist hochpreisigere Arzneimittel berücksichtigt werden, so wäre der Arzneimittelumsatz 2020 um 8,2\% gestiegen. Nur die rückläufigen Arzneimittelmengen und auch die sinkenden Preise im generischen Arzneimittelsegment und für erstmals verhandelte Erstattungsbeträge führten dazu, dass sich die Arzneimittelumsätze 2020 insgesamt nur um 4,9\% erhöht haben. Hochpreisige Arzneimittel, die $1.000 €$ und mehr kosten, machen mittlerweile rund $43 \%$ des gesamten ambulanten Umsatzes aus und haben Einzug in eine Vielzahl von Indikationsgruppen gehalten. Es ist zu vermuten, dass sich diese hohen Preise auf das Preisniveau zukünftiger Therapien in diesen Anwendungsgebieten als Preisanker auswirken werden.

Aber neben der ambulanten Versorgung sollte auch die stationäre Versorgung nicht aus dem Blick geraten. Gerade die Anwendung von neuen, komplexen und hochpreisigen Arzneimitteln erfolgt meist in dafür spezialisierten Krankenhäusern und bleibt zumeist bei der Ermittlung der Arzneimittelausgaben außen vor. Zunehmend gewichtigere Ausgabenposten dürfen jedoch bei der Diskussion über steigende Preise und Kosten nicht vernachlässigt werden. Gerade bei den Fragen rund um die Versorgung mit und Finanzierung von hochpreisigen Arzneimitteln sollten die Kran- 
kenhäuser den gesetzlichen Krankenkassen mehr Transparenz ermöglichen und die realen Arzneimittelkosten systematisch getrennt und vollständig mit ausweisen.

Die weltweit gewinnstärksten Pharmakonzerne haben ihre Geschäftsfelder insbesondere auf patentgeschützte und hochpreisige Arzneimittel konzentriert. Hier nutzen somit Konzerne die Möglichkeit, mit hohen Arzneimittelpreisen auch hohe Gewinne zu erzielen.

\section{Literatur}

Bausch J (2016) Innovations- und Kostenexplosion. KVH Aktuell 2016:22-25

Cassel D, Ulrich V (2020) Neue Arzneimittel im Visier der Kostendämpfung - Mythen und Fakten zur Ausgabenentwicklung in der GKV-Arzneimittelversorgung. RPG Recht Polit Im Gesundheitswes 26(1):13-27

Deutsche Apothekerzeitung (2016) Herausforderung Hochpreiser. https://www.deutsche-apothekerzeitung.de/daz-az/2016/az-6-2016/herausforderunghochpreiser. Zugegriffen: 20. Juli 2021

DiMasi JA, Hansen RW, Grabowski HG, Lasagna L (1991) Cost of innovation in the pharmaceutical industry, $\mathrm{J}$ Health Econ 10(2):107-142

DiMasi JA, Grabowski HG, Hansen RW (2016) Innovation in the pharmaceutical industry: new estimates of R\&D costs, J Health Econ 47(1):20-33

DiMasi JA, Hansen RW, Grabowski HG (2003) The price of innovation: new estimates of drug development costs. J Health Econ 22(2):151-185

EY (2019) Top 500 F\&E: Wer investiert am meisten in Innovationen? https://assets.ey.com/content/dam/ ey-sites/ey-com/de_de/news/2019/07/ey-top-500-feunternehmen-der-welt-2019.pdf?download. Zugegriffen: 5. Juli 2021

EY (2021) Die größten Pharmafirmen weltweit. Analyse der wichtigsten Finanzkennzahlen der Geschäftsjahre 2018, 2019 und 2020. https://assets.ey.com/ content/dam/ey-sites/ey-com/de_de/news/2021/06/ ey-pharma-bilanzen-2021.pdf?download. Zugegriffen: 5. Juli 2021

Glaeske G (2016) Zwischen Kosteneffektivität und „Mondpreisen“ - Zur Preisdiskussion auf dem Pharmamarkt. Implicon Plus 06:1-11
Greiner W, Witte J, Gensorowsky D et al (2020) AMNOGReport 2020. 10 Jahre AMNOG - Rückblick und Ausblick. Beiträge zur Gesundheitsökonomie und Versorgungsforschung, Bd. 32

Haas A, Tebinka-Olbrich A, Zentner A et al (2019) Ergebnisse des AMNOG-Erstattungsbetragsverfahrens. In: Schwabe U, Paffrath D, Ludwig WD et al (Hrsg) Arzneiverordnungs-Report 2019. Springer, Berlin

Kerpel-Fronius S, Baroutsou V, Becker S et al (2020) Development and use of gene therapy orphan drugs - ethical needs for a broader cooperation between the pharmaceutical industry and society. Front Med 7:1016

Korzilius H (2017) Hohe Preise gefährden die Versorgung. Dtsch Arztebl 113:49

Leclerque G, Mostert C (2021) Die Krankenhausbudgets 2018 und 2019 im Vergleich. Krankenhaus-Report 2021. Springer, Berlin

Lohmüller J, Schröder M, Telschow C (2019) Der GKVArzneimittelmarkt 2018: Trends und Marktsegmente. In: Schwabe U, Paffrath D, Ludwig WD (Hrsg) Arzneiverordnungs-Report 2019. Springer, Berlin Heidelberg, S 249-299

Morgan SG, Bathula HS, Moon S (2020) Pricing of pharmaceuticals is becoming a major challenge for health systems. BMJ 368:1-4

Mühlbauer B (2017) Mondpreise - oder was? Arzneiverordn Prax 44:56-57

Richard S (2016) Neue Regeln im Pillenpoker. Gesundh Ges 16(3):32-37

Sanger-Katz M (2014) \$1,000 Hepatitis Pill Shows Why Fixing Health Costs Is So Hard. The New York Times, 02.08.2014. https://www.nytimes.com/2014/ 08/03/upshot/is-a-1000-pill-really-too-much.html. Zugegriffen: 8. Aug. 2021

Sarrazin C, Zimmermann T, Berg T et al (2018) S3Leitlinie „Prophylaxe, Diagnostik und Therapie der Hepatitis-C-Virus (HCV)-Infektion“ AWMFRegister-Nr.: 021/012; Z Gastroenterol 2018; 56: 756-838. https://www.awmf.org/uploads/tx_ szleitlinien/021-0121_S3_Hepatitis-C-Virus_HCVInfektion_2018-07.pdf. Zugegriffen: 19. Juli 2021

Schlander M, Hernandez-Villafuerte K, Cheng CY, Mestre-Ferrandiz J, Baumann M (2021) How Much Does It Cost to Research and Develop a New Drug? A Systematic Review and Assessment, PharmacoEconomics, 1-27

WIdO (2021) Methodische Erläuterungen und ergänzendes Material zur Arzneimittelmarktanalyse. https:// wido.de/forschung-projekte/arzneimittel/methoden/ 
Open Access Dieses Kapitel wird unter der Creative Commons Namensnennung 4.0 International Lizenz (http:// creativecommons.org/licenses/by/4.0/deed.de) veröffentlicht, welche die Nutzung, Vervielfältigung, Bearbeitung, Verbreitung und Wiedergabe in jeglichem Medium und Format erlaubt, sofern Sie den/die ursprünglichen Autor(en) und die Quelle ordnungsgemäß nennen, einen Link zur Creative Commons Lizenz beifügen und angeben, ob Änderungen vorgenommen wurden.

Die in diesem Kapitel enthaltenen Bilder und sonstiges Drittmaterial unterliegen ebenfalls der genannten Creative Commons Lizenz, sofern sich aus der Abbildungslegende nichts anderes ergibt. Sofern das betreffende Material nicht unter der genannten Creative Commons Lizenz steht und die betreffende Handlung nicht nach gesetzlichen Vorschriften erlaubt ist, ist für die oben auf geführten Weiterverwendungen des Materials die Einwilligung des jeweiligen Rechteinhabers einzuholen. 